\title{
CHANGES IN THE EXPRESSION OF DNA METHYLATION RELATED GENES IN LEUKOCYTES OF PERSONS WITH ALCOHOL AND DRUG DEPENDENCE
}

\author{
M. Krasteva, Y. Koycheva, T. Taseva, S. Simeonova \\ Institute of Plant Physiology and Genetics, Laboratory of Genome Dynamics and Stability, \\ Bulgarian Academy of Sciences - Sofia, Bulgaria
}

\begin{abstract}
Background and objectives. Though numerous studies have shown that the dysregulation of the epigenetic control is involved in disease manifestation, limited data is available on the transcriptional activity of DNA methylation related genes in alcohol and drug addiction. With regard to this, in this study we analyzed the expression levels of genes involved in DNA methylation, including DNMT1, DNMT3a, MeCP2, MBD1, MBD2, MBD3 and MBD4, in blood samples of alcohol and drug dependent persons in comparison to healthy abstainers. Methods. The study included 51 participants: 16 persons with alcohol dependence, 17 persons with drug dependence and 18 clinically healthy controls. To detect the relative mRNA expression levels of the studied genes, Quantitative reverse transcription polymerase chain reaction (qRT-PCR) analysis was applied. Results. Of the seven studied genes, four showed altered expression. MeCP2 and MBD1 were downregulated in the alcohol dependent group ( $F C=0.805, p=0.015$ and $F C=0.846, p=0.034$, respectively), while DNMT1 and MBD4 were upregulated in the group with drug dependence $(F C=1.262, p=0.001$ and $F C=1.249, p=0.005$, respectively). No statistically significant changes in the relative mRNA expression were found for DNMT3a, MBD2 and $M B D 3$ genes. Conclusions. Our results are indicative for a role of DNA methylation related genes in alcohol and drug addiction mediated through changes in their transcriptional activity. Studies in this direction will enable better understanding of the underlying mechanisms of addictions supporting the development of more effective therapeutic strategies.
\end{abstract}

Key words: DNA methylation; Gene expression; mRNA, Alcohol dependence; Drug dependence

Corresponding author: Maria E. Krasteva, Laboratory of genome dynamics and stability, Institute of plant physiology and genetics, Bulgarian Academy of Sciences, 21 Acad. G. Bonchev Str., 1113 Sofia, Bulgaria; tel: +359 (2) 979 2678; e-mail: maria_krasteva@abv.bg

\section{INTRODUCTION}

rug and alcohol addiction is a detrimental neuropsychiatric disorder, which involves a progression from impulsive to compulsive substance consuming behavior resulting in prolonged and relapsing use. According to WHO in 2017 about 35 million are affected by drug use disorders and about 585000 people have died as a result of drug use. At the same time alcohol consumption accounts for more than 3000000 deaths every year and the use of alcohol is considered to be a major factor in the development of more than 200 diseases and injuries. 
Studies have shown that the predisposition to alcohol and drug addiction is a combination of genetic and environmental factors. On a molecular level such cooperation can manifest through epigenetic mechanisms [1]. Epigenetics is "the structural adaptation of chromosomal regions so as to register, signal or perpetuate altered activity states" [2]. The best studied epigenetic modification is DNA methylation, which in eukaryotes is mainly limited to cytosine bases. There are two mechanisms through which DNA methylation can silence genes. It can either prevent association of DNA-binding factors to their target sequences or can recruit repressive chromatin proteins to induce transcriptional repression [3].

The methylation of the cytosine to 5-methyl cytosine is catalyzed by a group of enzymes called DNA methyltransferases (DNMTs). Two classes can be distinguished: de novo methyltransferases DNMT3a and DNMT3b, which introduce methylation to previously unmethylated DNA and maintenance methyltransferase DNMT1, which catalyses the methylation of the newly synthesised DNA strand during the process of replication [4]. Another group of proteins, methyl-CpG-binding domain proteins (MBDs, including MBD1-6 and MeCP2), selectively bind to methylated DNA and contribute to the inhibition of gene expression. All of them are important in neural development and plasticity and alteration in their function occur in many neurological disorders [5].

Studies have indicated that the dysregulation of the epigenetic control in the brain contributes to the neuropathological manifestations of several psychiatric disorders, including alcohol and drug addictions [69]. The brain has the highest overall level of DNA methylation and the highest genomic content of 5-hydroxymethylcytosine than any other human tissue [10], which is suggestive that the deregulation of DNA methylation/demethylation pathways is likely to play a crucial role in neurological function and disease.

Unusual methylation pattern has been observed in humans and animal models under alcohol and drugs exposure. Alcohol causes global DNA hypomethylation in the brain [11-13], though a study reported global increase in DNA methylation in blood cells of alcoholic patients undergoing early alcohol withdrawal [14]. A study on alcohol dependent persons and controls found no differences in total methylation; however, about $20 \%$ of all promoters were differentially methylated between the groups [15].

Regarding the potential for clinical application of blood samples, it is of particular interest to investigate the correlation between brain and blood DNA methylation profiles. A study showed that abnormali- ties in the methylation machinery found in the brain of patients with schizophrenia are also present in their lymphocytes [16], supporting the hypothesis of a common epigenetic dysregulation.

The transcription activity of DNA methylation related genes in persons with substance use disorders is limitedly studied. In view of this, here we evaluated the expression pattern of genes implicated in DNA methylation, including the methyltransferases DNMT1 and $D N M T 3 a$, and the methyl $\mathrm{CpG}$ binding domain proteins MeCP2, MBD1, MBD2, MBD3 and MBD4 in blood samples of alcohol and drug dependent persons in comparison to healthy abstainers.

\section{MATERIALS AND METHODS}

\section{Participants}

A total of 51 persons were included in this study: 1) 16 persons diagnosed with alcohol dependence who were undergoing treatment at the State psychiatric hospital for treatment of drug and alcohol addictions in Sofia (27-57 years; mean $43 \pm 9$ years; males to females $10 / 6)$; 2) 17 persons with drug dependence who are under treatment at two clinical centres in Sofia (27-62 years, mean $41 \pm 8$; males to females $12 / 5)$; 3) 18 clinically healthy abstainers, who were matched as closely as possible to alcohol and drug dependent groups according to age and gender.

Diagnosis of alcohol and drug dependent people was done using the ICD-10 diagnostic system according to their medical records. All participants completed a questionnaire concerning their socio-demographic status. All persons included in the study were adults and gave written informed consents for participation in the study and collection of data. To obtain RNA specimens, the participants donated a blood sample of 5-6 ml. The collected blood samples were transferred to the laboratory and processed within 2 hours to avoid RNA degradation.

The study was carried out in accordance with the Declaration of Helsinki and was approved and conducted under the regulation of the Local Research Ethical Committee (1/20.03.2019).

\section{RNA isolation and cDNA synthesis}

To isolate total RNA, QiAamp RNA Blood mini kit (Qiagen) was used following the manufacturer's recommendations. To eliminate any potential genomic DNA contamination, an optional step was added (RNase-free DNase set, Qiagen). The quality and quantity of RNA samples were determined by spectrophotometrical (BioSpec-nano Spectrophotometer - Shimadzu Biotech) and electrophoretical (1.5\% 
agarose gel in $1 \times T A E$ ) assessment. Only high quality isolates (absorption ratio $260 / 280 \mathrm{~nm}>1.8$ ) were used for analysis.

An aliquot of $500 \mathrm{ng}$ RNA obtained from each sample was used to synthesize complementary DNA (cDNA). FIREScript RT cDNA Synthesis Mix (Solis Biodyne) was used and manufacturer's protocol was followed. As negative controls, two reactions were used: one missing reverse transcriptase and one missing RNA template. The synthesized cDNA was stored longterm at $-30^{\circ} \mathrm{C}$.

\section{qRT-PCR analysis}

To study the expression patterns, Quantitative reverse transcription polymerase chain reaction (qRTPCR) analysis was applied. The synthesized cDNA was used as a template to detect the relative mRNA expression levels of DNMT1, DNMT3a, MeCP2, $M B D 1, M B D 2, M B D 3$ and MBD4 genes in the three studied groups. HOT FIREPol EvaGreen qPCR Mix Plus (no ROX) (Solis Biodyne) kit was used. To normalize the target gene expression, the housekeeping gene ATP $5 b$ was used as a reference gene. The primers used for amplification are according to literature data $[17,18]$.

The reaction was performed in $10 \mu$ volume containing: 1x qPCR Mix, $0.3 \mu \mathrm{M}$ of each primer set, DNaseRNaseFree water and $1 \mu$ of 5 -fold diluted cDNA on PikoRealTM Real-Time PCR System (Thermo Fisher Scientific Inc.). The cycling conditions included 15 min at $95^{\circ} \mathrm{C}$, followed by 40 cycles of $15 \mathrm{sec}$ at $95^{\circ} \mathrm{C}$, $30 \mathrm{sec}$ at $60^{\circ} \mathrm{C}, 45 \mathrm{sec}$ at $72^{\circ} \mathrm{C}$, and final elongation for 5 min at $72^{\circ} \mathrm{C}$. Non template control (NTC) was added in each experiment. Data was analyzed using PikoReal Software version 2.1 (Thermo Fisher Scientific Baltics UAB). Standard curves were generated to assess the efficiency of the amplification.

\section{Data analysis}

Relative quantification and statistical evaluation of gene expression data was performed using the Relative Expression Software Tool (REST) [19]. Values of $p$ below 0.05 were considered statistically significant.

\section{RESULTS}

Relative expression of DNA methylation related genes in people with alcohol dependence (AD), drug dependence (DD) and controls (C).

\section{DNMT1}

We found that the relative expression of DNMT1 in people with drug dependence was upregulated compared to controls (DD vs $C, F C=1.262, p=0.001$,
Fig. 1a). Fold change was above the average in $35.29 \%$ of the cases reaching a maximum value of 1.728. No significant difference was observed in the alcohol dependence group compared to controls (AD vs $C, F C=1.084, p=0.240$, Fig. 1a).

\section{DNMT3a}

DNMT3a showed no significant difference in relative expression either in alcohol or drug dependence group when compared to controls $(A D$ vs $C, F C=$ $0.865, p=0.245 ;$ DD vs $C, F C=0.977, p=0.883$, Fig. 1b).

\section{MeCP2}

The relative expression level of $M e C P 2$ was decreased in the alcohol dependence group compared to the controls ( $A D$ vs $C, F C=0.805, p=0.015$, Fig. 1c). In $43.75 \%$ of the cases, MeCP2 fold change was below the average reaching a minimal value of 0.420 . No significant deference in the relative MeCP2 expression was observed in the drug dependence group compared to the controls (DD vs $\mathrm{C}, \mathrm{FC}=1.042, \mathrm{p}=$ 0.547, Fig. 1C).

\section{MBD1}

$M B D 1$ relative expression was downregulated in the alcohol dependence group compared to the controls ( $A D$ vs $C, F C=0.846, p=0.034$, Fig. $1 d$ ). In $37.5 \%$, MBD1 mRNA level was below the average reaching a minimal value of 0.566 . No significant changes in mRNA transcription level of MBD1 were observed in the drug dependent group compared to the controls (DD vs C, FC = 1.121, $p=0.195$, Fig. 1d).

\section{MBD2}

The relative expression of $M B D 2$ in the alcohol and drug dependence group was not different in comparison with controls (AD vs $C, F C=0.969, p=0.698$, DD vs $C, F C=1.030, p=0.715$, Fig. $1 e$ ).

\section{MBD3}

$M B D 3$ relative expression of in the alcohol and drug dependence group was comparable to this in the controls (AD vs $C, F C=1.021, p=0.306$, DD vs $C$, $F C=1.080, p=0.798$, Fig. 1f).

\section{MBD4}

In the drug dependent group MBD4 relative expression was upregulated compared to the controls (DD vs $C, F C=1.249, p=0.005$, Fig. $1 \mathrm{~g}$ ). In $47 \%$ of the samples MBD4 mRNA level was above the average, reaching a maximum value of 2,102 . MBD4 transcription levels in the alcohol dependent group did not differ significantly compared to the controls ( $A D$ vs $C$, $F C=0.876, p=0.268$, Fig. 1g). 
a

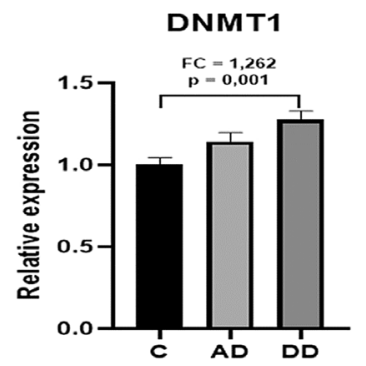

c

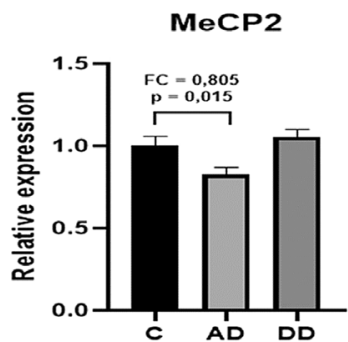

e

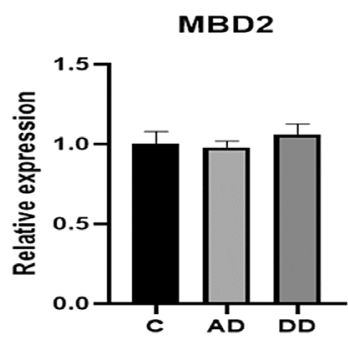

b

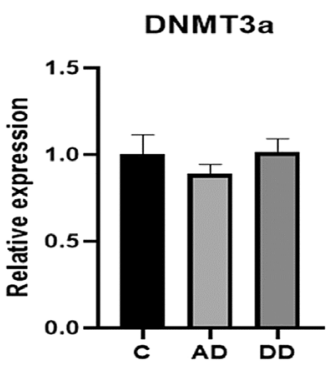

d

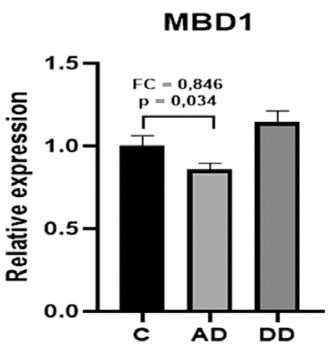

f

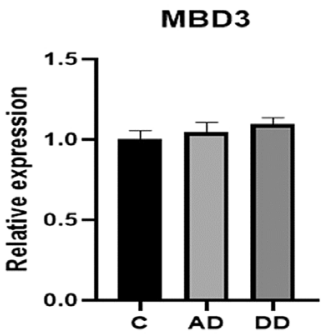

g

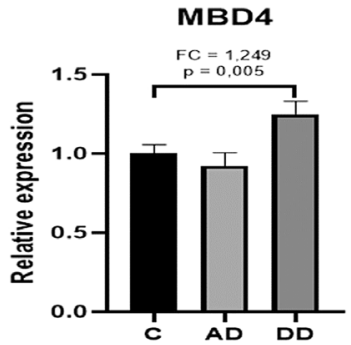

Fig. 1. Relative mRNA expression of a - DNMT1 gene in persons with alcohol (AD) and drug dependence (DD) vs. controls (C); $\mathbf{b}-$ DNMT3a gene in persons with alcohol (AD) and drug dependence (DD) vs. controls (C); $\mathbf{c}-M e C P 2$ gene in persons with alcohol (AD) and drug dependence (DD) vs. controls (C); $\mathbf{d}-M B D 1$ gene in persons with alcohol (AD) and drug dependence (DD) vs. controls (C); $\mathbf{e}-M B D 2$ gene in persons with alcohol (AD) and drug dependence (DD) vs. controls (C); $\mathbf{f}-M B D 3$ gene in persons with alcohol (AD) and drug dependence (DD) vs. controls (C); $\mathbf{g}-M B D 4$ gene in persons with alcohol (AD) and drug dependence (DD) vs. controls (C). FC - fold change; $p$-values less than 0.05 were considered statistically significant

\section{DISCUSSION}

Until recently, a major focus in biomedical research was the study of sequence variations in the DNA leading to structural alterations in the resultant pro- teins. During the last decades, data have been accumulated indicating that epigenetic changes in the genetic material, other than the DNA sequence itself, may cause alterations in gene function, which could be correlated with disease manifestation. Alteration 
in DNA methylation at promoters, enhancers or gene bodies has been described in a number of complex diseases including alcohol and drug addictions.

The alteration in DNA methylation pattern might be due to inhibition or activation of DNMTs and MBDs genes. These have a different role in interpreting DNA methylation patterns. While DNMTs are DNA methylation "writers" involved in establishing and maintaining methylation marks throughout development and differentiation, MBDs are the "readers", which act as translators between DNA methylation and histone modifications [20, 21].

DNMT1 enables the major maintenance methyltransferase activity, though it is suspected to participate in de novo DNA methylation as well [22]. The loss of DNMT1 activity in knockout mice results in decrease in global DNA methylation and death of embryos. The chronic alcohol exposure leads to downregulation of DNMT1 expression in the sperm and lower offspring weight in rats [23]. However, exposure to alcohol is also correlated with DNMT1 upregulation in NAs of mice accompanied by hypoacetylation of histone $\mathrm{H} 4$ [24]. This is consistent with our finding of a significant increase in DNMT1 transcription levels in patients with drug dependence, though such DNMT1 upregulation was not observed in the alcohol dependant group. The higher levels of DNMT1 transcrips are expected to result in increase in DNMT1 methyltransferase activity, which may lead to chromatin condensation and gene repression.

DNMT3a has an active role in de novo DNA methylation at the later embryo stages during development and in differentiated cells as well. However, it could also function as a maintenance DNA methyltransferase [25]. Miozzo et al. demonstrated that ethanol exposure leads to an increase in the levels of $D N$ MT3a transcripts and DNMT3a protein in mice [26]. Increased global DNA methylation in lymphocytes of patients with alcohol dependence was observed, despite the considerable (2.4 fold) decrease in $D N$ MT3a expression [27]. Acute alcohol exposure inhibits DNMT activity and leads to decrease in DNA methylation and DNMT3a levels in astrocytes [28]. In mice model of cocaine addiction, the expression of DNMT3a in the NAs was upregulated and these changes were apparent 3 weeks after the end of cocaine administration [29]. Though the above findings attribute a role of DNMT3a in alcohol and drug addictions, our study did not add support to this as no changes in DNMT3a relative expression were observed in the blood samples of the studied alcohol or drug dependent persons.
MeCP2 has an important role in chromatin remodelling and acts as a transcription repressor of specific genes, though it is suspected to exert more global effects over the genome. MeCP2 is critical in neural development and is highly expressed in neurons. A study reported increased levels of MeCP2 in dorsal striatal neurons in rats after cocaine administration [30]. Another study observed that cocaine administration increases MeCP2 expression in hippocampus, frontal cortex, and NAc of rats [31]. Deng et. al. confirmed a role of MeCP2 in drug addiction by studying the effects of another psychostimulant, amphetamine [32]. The conducted here analysis also implies a role of MeCP2 in addictions as confirmed by the observed decrease in MeCP2 expression in leukocytes of patients with alcohol dependence.

MBD1 is highly expressed in the neuronal stem cells and neurons. MBD1 knockout mice are viable and do not have severe neuronal defects, but show reduced hippocampal neurogenesis, altered spatial learning and increased genome instability [33]. In humans, mutations in MBD1 are found in sporadic cases of autism spectrum disorder [34]. Limited studies are available regarding the involvement of $M B D 1$ in substance use disorders. A study showed that after 10 days of i.p. injection of cocaine, MBD1 along with $M e C P 2$, is induced in the brain of rats, presumably leading to reduced transcription [35]. Such a correlation in the expression of $M B D 1$ and MeCP2 was found also in our study in the alcohol dependence group though in the opposite direction: the expression level of MBD1 is reduced along with a similar decrease in the expression of MeCP2.

MBD2 knockout mice are viable and show mild pup nurturing deficits and hypoactivity. Interestingly MBD2 can target the NuRD repressive complex not only to the methylated $\mathrm{CpGs}$, where it mediates repression of the local chromatin, but also to unmethylated $\mathrm{CpGs}$, where promotes activation [36]. Here we detected no statistically significant difference in the expression of $M B D 2$ in the alcohol or drug dependent groups, which is in agreement with the results from a study of patients with chronic alcoholism [27].

MBD3 is also part of the NuRD repressive complex. MBD3 knockout leads to embryonic lethality in mice. It is highly expressed in embryonic stem cells and somatic tissues. Several studies show upregulation of $M B D 3$ in postmortem brain samples of alcoholics and cocaine abusers [12, 37, 38]. However, our results fail to detect an alteration in the expression level of $M B D 3$ in the blood of alcohol and drug dependent patients. 
MBD4 is unique among the family members with its C-terminal glycosylase motif, which enables mismatch repair of methylated and unmethylated CpGs. It is highly expressed in embryonic stem cells and somatic tissues. Deletion of MBD4 is not lethal, but these mice show increase in CpG to TpG transitions. MBD4 frequently cooperates with DNMT1 to repress gene expression. In 293T cells the expression of CDKN1A/p21 was increased upon knockdown of MBD4 and DNMT1 and the combined knockdown produced the most pronounced effect with 30 fold increase [39]. The same study shows that the combined effect of MBD4 and DNMT1 depletion leads to 100 fold induction of the $\mathrm{MSH} 4$ gene, despite the fact that the MSH4 promoter was still methylated. Another study shows that MBD4 and DNMT1 bind to Gitr promotor and their overexpression significantly inhibits Gitr expression [40]. On the other hand MBD4 expression was not significantly different in DNMT1 positive neurons of psychotic patient with history of alcohol abuse [41]. In our study the expression of MBD4 was increased in the drug dependent group. This increase is probably connected to the observed upregulation of DNMT1 and a possible subsequent hypermethylation as opioids are shown to induce DNA hypermethylation [42].

\section{CONCLUSION}

In conclusion, of the seven studied here genes, four showed altered expression in blood samples of patients with alcohol and drug dependence. MeCP2 and $M B D 1$ were downregulated in the alcohol dependent group, while DNMT1 and MBD4 were upregulated in the group with drug dependence. In addition to other studies in animal models and human, our results are indicative for a role of epigenetic DNA methylation in substance use disorders mediated through changes in DNMTs and MBDs activity. Studies in this direction will contribute to a better understanding of addiction mechanisms and are expected to support the development of more effective therapeutic strategies.

\begin{abstract}
Acknowledgements: The authors are grateful to Rositsa Racheva, PhD, Tsveta Raycheva, MD and Emiliya Nikolova for taking part in the collection of the participants included in this study. This work was supported by the Bulgarian National Science Fund, Ministry of Education and Science, Bulgaria, grant number KP-06-H25/5 "Correlation between psychosocial and epigenetic characteristics in problematic psychoactive substances use". The authors Maria Krasteva and Yana Koycheva contributed equally to this work.
\end{abstract}

Disclosure summary: The authors have nothing to disclose.

\section{REFERENCES}

1. Nielsen D, Utrankar A, Reyes $\mathrm{J}$ et al. Epigenetics of drug abuse: Predisposition or response. Pharmacogenomics, 2012, 13, 1149-1160.

2. Bird A. Perceptions of epigenetics. Nature, 2007, 447(7143), 396-398.

3. Klose RJ, Bird AP. Genomic DNA methylation: the mark and its mediators. Trends Biochem Sci, 2006, 31(2), 89-97.

4. Lyko F. The DNA methyltransferase family: a versatile toolkit for epigenetic regulation. Nat Rev Genet, 2018, 19(2), 81-92.

5. Du Q, Luu PL, Stirzaker C, Clark SJ. Methyl-CpG-binding domain proteins: readers of the epigenome. Epigenomics, 2015, 7(6), 1051-1073.

6. Houston I, Peter CJ, Mitchell A et al. Epigenetics in the human brain. Neuropsychopharm, 2013, 38, 183-197.

7. Grayson DR, Guidotti A. The dynamics of DNA methylation in schizophrenia and related psychiatricdisorders. Neuropsychopharm, 2013, 38, 138-166.

8. Guidotti A, Auta J, Chen Y et al. Epigenetic GABAergic targets in schizophrenia and bipolar disorder. Neuropharm, 2011, 60, 1007-1016.

9. Mill J, Tang T, Kaminsky $Z$ et al. Epigenomic profiling reveals DNA-methylation changes associated with major psychosis. Am J Hum Genet, 2008, 82, 696-711.

10. Lister R, Mukamel EA. Turning over DNA methylation in the mind. Front Neurosci, 2015, 9, 252.

11. Garro AJ, McBeth DL, Lima V, Lieber CS. Ethanol consumption inhibits fetal DNA methylation in mice: implications for the Fetal Alcohol Syndrome. Alcohol Clin Exp Res, 1991, 15, 395-398.

12. Ponomarev I, Wang S, Zhang L et al. Gene coexpression networks in human brain identify epigenetic modifications in alcohol dependence. J Neurosci, 2012, 32, 1884-1897.

13. Shukla SD, Velazquez J, French SW et al Emerging role of epigenetics in the actions of alcohol. Alcoholism: Clin Exp Res, 2008, 32(9), 1525-1534.

14. Bonsch D, Lenz B, Reulbach $U$ et al. Homocysteine associated genomic DNA hypermethylation in patients with chronic alcoholism. J Neural Transm, 2004, 111, 1611-1616.

15. Manzardo AM, Henkhaus RS, Butler MG. Global DNA promoter methylation in frontal cortex of alcoholics and controls. Gene, 2012, 498(1), 5-12.

16. Auta J, Smith RC, Donga E et al. DNA-methylation gene network dysregulation in peripheral blood lymphocytes of schizophrenia patients. Schizophrenia Res, 2013, 150, 312-318.

17. Kala R, Shah HN, Martin SL, Tollefsbol TO. Epigenetic-based combinatorial resveratrol and pterostilbene alters DNA damage response by affecting SIRT1 and DNMT enzyme expression, including SIRT1-dependent $\mathrm{y}-\mathrm{H} 2 \mathrm{AX}$ and telomerase regulation in triple-negative breast cancer. BMC Cancer, 2015, 15, 672.

18. Müller C, Readhead C, Diederichs et al. Methylation of the cyclin A1 promoter correlates with gene silencing in somatic cell lines, while tissue-specific expression of cyclin A1 is methylation independent. Mol Cell Biol, 2000, 3316-3329.

19. Pfaffl MW. A new mathematical model for relative quantification in real-time RT-PCR. Nucleic Acids Res, 2001, 29(9), e45.

20. Moore LD, Le T, Fan G. DNA methylation and its basic function. Neuropsychopharmacology Reviews, 2013, 38, 23-38.

21. Baubec T, Ivánek R, Lienert F, Schübeler D. Methylation-dependent and - independent genomic targeting principles of the MBD protein family. Cell, 2013, 53(2), 480-492. 
22. Vertino PM, Yen RW, Gao J, Baylin SB. De novo methylation of $\mathrm{CpG}$ island sequences in human fibroblasts overexpressing DNA (cytosine-5-)-methyltransferase. Mol Cell Biol, 1996; 16(8), 4555-4565.

23. Bielawski DM, Zaher FM, Svinarich DM, Abel EL. Paternal alcohol exposure affects sperm cytosine methyltransferase messenger RNA levels. Alcohol Clin Exp Res, 2002, 26, 347-351.

24. Warnault ED, Levine A, Barak S, Ron D. Chromatin remodeling - a novel strategy to control excessive alcohol drinking. Transl Psychiatry, 2013, 3, e231.

25. Jones PA, Liang G. Rethinking how DNA methylation patterns are maintained. Nat Rev Genet, 2009, 10, 805-811.

26. Miozzo F, Arnould H, De Thonel A et al. Alcohol exposure promotes DNA methyltransferase DNMT3a upregulation through reactive oxygen species-dependent mechanisms. Cell Stress Chaperones, 2018, 23, 115-126.

27. Bönsch D, Lenz B, Fiszer R et al. Lowered DNA methyltransferase (DNMT-3b) mRNA expression is associated with genomic DNA hypermethylation in patients with chronic alcoholism. J Neural Transm, 2006, 113(9), 1299-1304.

28. Zhang X, Kusumo H, Sakharkar AJ et al. Regulation of DNA methylation by ethanol induces tissue plasminogen activator expression in astrocytes. J Neurochem, 2014, 128(3), 344-349.

29. LaPlant Q, Vialou V, Covington HE 3rd, et al. DNMT3a regulates emotional behavior and spine plasticity in the nucleus accumbens. Nat Neurosci, 2010, 13(9), 1137-1143.

30. Im HI, Hollander JA, Bali P, Kenny PJ. MeCP2 controls BDNF expression and cocaine intake through homeostatic interactions with microRNA-212. Nat Neurosci, 2010, 13(9), 1120-1127.

31. Cotto B, Li H, Tuma RF, Ward SJ, Langford D. Cocaine-mediated activation of microglia and microglial MeCP2 and BDNF production. Neurobiol Dis, 2018, 117, 28-41.

32. Deng JV, Rodriguiz RM, Hutchinson AN et al. MeCP2 in the nucleus accumbens contributes to neural and behavioral responses to psychostimulants. Nat Neurosci, 2010, 13(9), 1128-1136.
33. Allan AM, Liang $X$, Luo $Y$, et al. The loss of methyl-CpG binding protein 1 leads to autism-like behavioral deficits. Hum Mol Genet, 2008, 17(13), 2047-2057.

34. Li H, Yamagata T, Mori M, Yasuhara A, Momoi MY. Mutation analysis of methyl-CpG binding protein family genes in autistic patients. Brain Dev, 2005, 27(5), 321-325.

35. Cassel S, Carouge D, Gensburger C, et al. Fluoxetine and cocaine induce the epigenetic factors MeCP2 and MBD1 in adult rat brain. Mol Pharmacol, 2006, 70(2), 487-492.

36. Pan H, Bilinovich SM, Kaur P et al. CpG and methylationdependent DNA binding and dynamics of the methylcytosine binding domain 2 protein at the single-molecule level. Nucleic Acids Res, 2017, 45, 9164-9177.

37. Zhou Z, Yuan Q, Mash DC, Goldman D. Substance-specific and shared transcription and epigenetic changes in the human hippocampus chronically exposed to cocaine and alcohol. Proc Natl Acad Sci U S A, 2011, 108, 6626-6631.

38. Liu J, Lewohl JM, Harris RA et al. Patterns of gene expression in the frontal cortex discriminate alcoholic from nonalcoholic individuals. Neuropsychopharmacology: Official Publication of the American College of Neuropsychopharmacology, 2006, 31(7), 1574-1582.

39. Laget S, Miotto B, Chin HG et al. MBD4 cooperates with DNMT1 to mediate methyl-DNA repression and protects mammalian cells from oxidative stress. Epigenetics, 2014, 9(4), 546-556.

40. Wang S, Li Y, Zhu F et al. DNMT1 cooperates with MBD4 to inhibit the expression of Glucocorticoid-induced TNFRrelated protein in human T cells. FEBS Lett, 2017, 591(13), 1929-1939.

41. Guidotti A., Dong E., Gavin D et al. DNA Methylation/Demethylation Network Expression in Psychotic Patients with a History of Alcohol Abuse. Alcoholism, clinical and experimental research, 2012, 37.

42. Knothe C, Doehring A, Ultsch A, Lötsch J. Methadone induces hypermethylation of human DNA. Epigenomics, 2016, $8(2), 167-179$.

Revised: 26 September 2020, Accepted: 3 October 2020 